

\section{INDICE}

EDITORIAL PERSONAL ÁMBITOS

Apresentação do monográfico. Abordagem qualitativa: olhares e práticas transdisciplinares nas ciências antropossociais

Presentation of the monograph. Qualitative approach: transdisciplinary views and practices in anthroposocial sciences

Ronaldo Nunes Linhares, António Pedro Costa

MONOGRAFICOS MONOGRAPHS

Identidades femininas na rede: as crianças falam!

Female identities on line: children can speak

Marta Maria Azevedo Queiroz

$12-31$

Transição de cuidados de enfermagem: ISBAR na promoção da segurança dos

doentes - revisão scoping

Transition of nursing care: ISBAR in promoting patient safety - scoping review

Ana Rita Esteves Figueiredo, Teresa Maria Ferreira dos Santos Potra, Pedro Ricardo Martins Bernardes Lucas

$32-48$

Integración de elementos cualitativos y cuantitativos en metodología observacional

Integration of qualitative and quantitative elements in observational methodology

M. Teresa Anguera, Angel Blanco-Villaseñor, José Luis Losada, Pedro Sánchez-Algarra

49-70

Atos educativos com oficines de ecografias: uma investigação otobiográfica

Educational acts at echographie's cineliers: an otobiographic research

Silas Borges Monteiro, Anaise Avila Severo

Actuación de las políticas: política como texto y política como discurso

Action of policies: policy as text and policy as discourse

Mónica Rocío Barón

88-104 


\section{ÁMBITOS PERSONALES PERSONAL ÁMBITOS}

Un retrato de la cultura local a través del Periodismo cultural. Análisis comparado de Sevilla y Porto Alegre

A portrait of the local culture through cultural Journalism. Comparative analysis of Seville and Porto Alegre

\section{ARTÍCULOS ARTICLES}

Microsociología del profesor universitario

Microsociology of an university professor

Antonio Fernández Vicente

La pobreza y el discurso de los mass media. Un estudio de la prensa local argentina

Poverty and mass media 's discourse. A study of the Argentine local press

María del Rosario Sanchez, Silvia London

La comunicación no verbal en las elecciones andaluzas de 2018. Comparativa de Susana Díaz y Teresa Rodríguez en el debate de RTVE

Non-verbal communication in the Andalusian municipal elections of 2018. Comparison of Susana Díaz and Teresa Rodríguez in the electoral RTVE debate

María Hernández Herrarte, Patricia Zamora-Martínez

El infoentretenimiento en la televisión de pago, Movistar+ y el canal \#0.

El uso transmedia de sus contenidos de humor

Infotainment on pay television, Movistar+ and channel \# 0 . The transmedia use of its humorous content

Metodología y formación docente cuestiones claves para la integración de las TIC en la educación

Methodology and teacher training as a key issue for ICTs integration in Education 


\section{RESEÑAS REVIEWS}

Aquelarre. Mujeres en la cultura de masas

Coven. Women in mass culture

Regla Ismaray Cabreja Piedra

216-220

Transición ecosocial y principios éticos en el periodismo: una guía para la comunicación de nuevas narrativas

The Eco-social transition and ethical principles in journalism: a guide for the communication of new narratives

Amanda Salazar Torres

Narrativas ecofeministas y mapa de transición ecosocial para medios de comunicación Eco-feminist narratives and ecosocial transition map for the media 


\title{
Transição de cuidados de enfermagem: ISBAR na promoção da segurança dos doentes - revisão scoping
}

\author{
Transition of nursing care: ISBAR in promoting patient safety - \\ scoping review
}

\begin{abstract}
Ana Rita Esteves Figueiredo, Escola Superior de Enfermagem de Lisboa (ESEL) Avenida Dom João II, n. 4.69.01, 1990-096. Lisboa. Portugal. anaritafigueiredo93@gmail.com | Orcid: https://orcid.org/0000-0002-5373-5284
\end{abstract}

Teresa Maria Ferreira dos Santos Potra, Escola Superior de Enfermagem de Lisboa (ESEL) Avenida Dom João II, n. 4.69.01, 1990-096. Lisboa. Portugal. tssantos@esel.pt | Orcid. https://orcid.org/ 0000-0002-5344-0136

Pedro Ricardo Martins Bernardes Lucas, Escola Superior de Enfermagem de Lisboa (ESEL) Avenida Dom João II, n. 4.69.01, 1990-096. Lisboa. Portugal. prlucas@esel.pt | Orcid. https://orcid.org/0000-0002-2560-7306

DOI: https://dx.doi.org/10.12795/Ambitos.2020.i49.03

\begin{abstract}
Resumo
O tema das transferências de doentes tem vindo a ganhar uma crescente valorização ao nível nacional e internacional nomeadamente com a proliferação de literatura referente à necessidade de melhorias na transição de cuidados de saúde. A evidência científica demonstra que uma comunicação eficaz entre os profissionais de saúde promove a segurança dos doentes. A comunicação eficaz pode ser conseguida através da utilização de ferramentas estruturadas que orientam a transferência de informação sendo frequentemente recomendada a utilização da técnica ISBAR
\end{abstract}


(Identificação; Situação; Background; Avaliação; Recomendações) durante o processo de transmissão de informação. O objetivo é: mapear e examinar a evidência científica relacionada com as vantagens da utilização da técnica ISBAR (Identificação, Situação, Antecedentes, Avaliação, Recomendações), pelos enfermeiros, na transmissão de informação em contexto hospitalar. Para tal, foi realizada uma pesquisa em três fases de acordo com a metodologia do Instituto Joanna Briggs (2015). 1) Pesquisa na MEDLINE e CINAHL, seguindo-se uma análise do título, resumo e termos indexados; 2) As palavras-chave e termos indexados foram pesquisados nas restantes bases de dados incluídas. 3) Pesquisa da lista de referências bibliográficas de todos os artigos incluídos. Os resultados demonstram que a utilização desta técnica permite melhorar a transferência de informação, evita lacunas, salvaguarda a segurança do doente. A transferência torna-se atempada e eficiente. A hierarquia profissional é nivelada, minimizando pressupostos. Conclui-se que a utilização da técnica ISBAR melhorou a transmissão de informação sobre os motivos da transferência e condição do doente, promovendo simplicidade, clareza e segurança durante a transmissão de informação clínica.

\section{Abstract}

The issue of patient safety during transfers has been gaining increasing importance at national and international levels, particularly with the proliferation of literature on the need for improvements in the health care transition. The scientific evidence shows that effective communication among health professionals increases patient safety. Effective communication can be achieved through the use of structured tools that guide the transfer of information, such as ISBAR technique (Identification, Current Situation, Background, Assessement, Recommendations), which is often recommended for the information transmission process'. Our objective is map out and analyse the scientific evidence related to the advantages of using the ISBAR technique in the transitions, by nurses, in hospital settings. It was conducted a three-step search strategy according to the Joanna Briggs Institute (2015). 1) A search of MEDLINE and CINAHL was undertaken, followed by an analysis of the words contained in the title, abstract and the index terms; 2) The keywords and index terms were searched across all the included databases; 3) Search of the reference lists of all the included articles. Results show that ISBAR improves the transfer of information and safeguards patient safety, as well as the handover information. The transfer is performed efficiently and timely. Professionals' hierarchy is leveled and assumptions are minimized. Concluding the use of ISBAR has improved the handover about patient's reasons for transfer and condition, promoting simplicity, clarity and safety during clinical transfers. 
Palavras-chave: Comunicação, Handoff, Hospital, ISBAR, Cuidados de Enfermagem Keywords: Communication, Handoff, Hospital, ISBAR, nursing care

\section{INTRODUÇÃO}

O tema das transferências de doentes tem vindo a ganhar uma crescente valorização ao nível nacional e internacional nomeadamente com a proliferação de literatura referente à necessidade de melhorias na transição de cuidados de saúde. A evidência científica demonstra, segundo Figueiredo, Potra \& Lucas (2019), que a existência de uma comunicação eficaz, aquando da transição de cuidados de saúde, é fundamental para assegurar a segurança do doente. A comunicação eficaz entre profissionais de saúde trata-se da transmissão de informação entre os profissionais, que se distingue por ser oportuna, precisa, completa, sem ambiguidade, atempada e compreendida pelo recetor (DGS, 2017).

A existência de uma comunicação eficaz entre os profissionais de saúde permite diminuir as situações de redundância, poupar tempo, aumentar a segurança do doente e a satisfação da equipa de saúde (Martin \& Ciurzynsky, 2015). Uma das estratégias que contribui para garantir a existência de uma comunicação eficaz é a utilização de uma ferramenta que promova a uniformização da transmissão de informação.

Assim, no âmbito da segurança da comunicação, em Portugal, a Direção Geral de Saúde em fevereiro de 2017 emitiu uma nova norma de orientação clínica, relacionada com a Comunicação eficaz na transição de cuidados de saúde. Nesta norma consta que a transição de cuidados deve ser realizada com recurso a uma comunicação eficaz na transferência de informação entre as equipas prestadoras de cuidados, para segurança do doente, devendo ser padronizada utilizando a técnica ISBAR (DGS, 2017).

O ISBAR trata-se de uma técnica que permite padronizar a comunicação em saúde sendo reconhecida por promover a segurança do doente em situações de transição de cuidados. A utilização desta técnica contribui para “(...) a rápida tomada de decisões, promove o pensamento crítico, diminui o tempo na transferência de informação e promove a rápida integração dos novos profissionais (...)" (DGS, 2017, p. 6).

A utilização da técnica ISBAR assume uma dimensão de escala mundial com diversos estudos desenvolvidos em vários pontos do globo com o objetivo de compreender de que forma é utilizada esta técnica, quais as suas vantagens e aplicabilidade nos diferentes contextos. Revisões Scoping sobre este tema são essenciais pois permitem sintetizar o conhecimento produzido sobre esta técnica cuja utilização tem vindo a ser 
recomendada por importantes instituições que trabalham no sentido de promover a segurança dos doentes.

Assim, considerando que a utilização desta técnica na transferência de cuidados é relativamente recente na literatura em enfermagem realizou-se a presente Scoping Review. O objetivo desta Scoping Review é mapear e examinar a evidência científica relacionada com as vantagens da utilização da técnica ISBAR, pelos enfermeiros, na transmissão de informação em contexto hospitalar.

A presente Scoping Review é baseada na metodologia do Instituto Joanna Briggs (Peters, Godfrey, Mclnerney, Munn, Tricco, \& Khalil, 2020). A questão da Scoping Review é: Quais as vantagens na utilização da técnica ISBAR, pelos enfermeiros, nas transições de cuidados de saúde em contexto hospitalar? Utilizou-se a estratégia PCC: População - Enfermeiros; Conceito - Transição de cuidados de saúde; ISBAR; Contexto - Hospital.

A pesquisa foi realizada de maio a junho de 2019 e levada a cabo sem limite temporal dado que se pretendeu abranger toda a literatura existente sobre o tema em análise. $O$ estudo incluiu todos os estudos em português, espanhol e inglês. Foi utilizada uma estratégia em três etapas: pesquisa preliminar sobre o tema utilizando as bases de dados CINAHL e MEDLINE. Para pesquisar revisões scoping foram utilizadas a JBI Database of Systematic Reviews and Implementation Reports, the Cochrane Library e Epistemonikos. Não foram encontradas revisões scoping sobre o tema. No motor de busca EBSCOhost, nas bases de dados CINAHL Plus full text e a MEDLINE with full texto, foram utilizados os termos: Enfermeiro (nurse) (População), transferências (handoff); ISBAR (Conceitos) e hospital (Contexto). Dos artigos obtidos foi realizada uma análise do título, resumo e termos indexados. Por fim foi realizada uma pesquisa da lista de referências bibliográficas de todos os artigos incluídos.

\section{REVISÃO TEÓRICA E PROBLEMÁTICA}

A problemática da segurança do doente tem vindo a ser amplamente estudada sendo que o relatório "To err is human" do Instituto de Medicina Americano publicado no ano 2000, foi o primeiro e grande impulsionador do movimento em torno da segurança do doente. O relatório, elaborado há cerca de 20 anos, alertava o mundo para a complexidade da medicina sendo esta uma área que envolve muitos riscos e que pode causar sofrimento, dano, incapacidade e mesmo a morte. Contudo esta área continua a ser estudada sendo que, a nível internacional, a segurança do doente tem vindo a ser abordada por inúmeras organizações tais como a Organização Mundial de Saúde (OMS), The Joint Commission, The Agency for Healthcare Research and Quality, e o Institute for Healthcare Improvement. 
A evidência científica demonstra que os erros médicos originaram até 400.000 mortes por ano em 2013, tornando a comunicação efetiva ainda mais importante, especialmente em áreas de alto risco como: medicinas, serviços de urgência e serviços de pediatria (Eppich, 2015).

Em Portugal não é conhecida a verdadeira extensão, nem as consequências, das lacunas de segurança relacionadas com a prestação de cuidados de saúde, contudo, de acordo com o estudo realizado por Silva, Pedrosa, Leça \& Silva (2016), por cada 100 internamentos hospitalares em 10 ocorrem complicações devido a um qualquer erro ou incidente.

Assim, a existência de uma comunicação eficaz entre os profissionais, aquando da transição de cuidados de saúde, é fundamental para que seja possível melhorar a segurança do doente.

A comunicação eficaz é entendida como a transmissão de "(...) informação entre os profissionais de saúde, que se caracteriza por ser oportuna, precisa, completa, sem ambiguidade, atempada e compreendida pelo recetor" (DGS, 2017, p. 4). A comunicação eficaz na área da saúde requer a existência de conhecimento, competência e empatia sendo que uma das estratégias que contribui para garantir a existência de uma comunicação mais eficaz é a utilização de uma ferramenta que permita uniformizar a transmissão de informação (Figueiredo et al., 2019). Ao nível dos planos nacionais, o Plano Nacional para a Segurança dos Doentes 2015-2020, fornece orientações para que os gestores do Serviço Nacional de Saúde em Portugal possam aplicar métodos, definir objetivos, e metas, com a finalidade de minimizar os riscos associados à prestação de cuidados de saúde.

Este plano, de forma a melhorar a prestação de cuidados e garantir a segurança dos doentes, recorre a diferentes objetivos estratégicos, entre os quais, o aumento da segurança da comunicação. No âmbito da segurança da comunicação a Direção Geral de Saúde, em fevereiro de 2017, emitiu uma nova norma relacionada com a comunicação eficaz na transição de cuidados de saúde afirmando que a “(...) transição de cuidados deve obedecer a uma comunicação eficaz na transferência de informação entre as equipas prestadoras de cuidados, para segurança do doente, devendo ser normalizada utilizando a técnica ISBAR (...)" (DGS, 2017, p. 1). O conceito de transição de cuidados de saúde diz respeito a:

Qualquer momento da prestação em que se verifique a transferência de responsabilidade de cuidados e de informação entre prestadores, que tem como missão a continuidade e segurança dos mesmos. São exemplos, a transição de cuidados entre os cuidados de saúde primários, os cuidados hospitalares e os cuidados continuados integrados, bem como, a transição intra/ inter instituições. 
São momentos vulneráveis/ críticos da transição de cuidados para a segurança do doente os momentos cuja complexidade envolvem um maior risco de erro na transferência de informação, como é o caso das admissões e altas hospitalares para o domicílio ou para outro nível de cuidados, e das mudanças de turno na mesma instituição (...) (DGS, 2017, p. 4).

A Joint Commission, no período de 2010-2013, realizou uma avaliação na qual evidenciou que as falhas de comunicação continuam a ser os eventos que mais potenciam a ocorrência de eventos adversos (Shapiro, 2017). No contexto nacional, de acordo com a Direção Geral de Saúde (2017), ao nível das falhas na comunicação em situações de transição de cuidados, é de referenciar um estudo realizado no ano de 2012, no qual em $50 \%$ dos casos analisados se verificou a inexistência de uma comunicação eficaz entre os profissionais de forma a garantir a continuidade informacional nos cuidados de saúde. Funk et al., (2016) refere que $80 \%$ dos erros médicos graves envolvem falhas de comunicação entre os cuidadores quando os doentes são transferidos. Neste sentido, um dos métodos de comunicação que é referenciado como sendo promotor de uma comunicação eficaz, e com maior segurança para o doente, está relacionado com técnica ISBAR.

O ISBAR trata-se de um instrumento que permite padronizar a comunicação em saúde, sendo uma técnica reconhecida por promover a segurança do doente em situações de transição de cuidados. Esta técnica permite, através de formas simples, memorizar construções complexas que são utilizadas na transmissão verbal de informação sobre o estado de saúde dos doentes, em que: (I) corresponde à identificação, (S) à situação atual, $(B)$ aos antecedentes, $(A)$ à avaliação, $(R)$ às recomendações (DGS, 2017). A utilização da técnica ISBAR é recomendada por inúmeras organizações de saúde, uma vez que é de fácil memorização, sendo possível utilizar em vários contextos, pois recorre a uma metodologia “(...) padronizada, simples, flexível, concisa e clara para comunicar informações relativas a esses cuidados (...)" (DGS, 2017, p. 6). Esta técnica “(...) contribui para a rápida tomada de decisões, promove o pensamento crítico, diminui o tempo na transferência de informação e promove a rápida integração dos novos profissionais (...)" (DGS, 2017, p. 6).

Assim, considerando que a utilização desta técnica na transferência de cuidados de enfermagem é relativamente recente na literatura em enfermagem, realizou-se a presente revisão scoping. 


\section{RESULTADOS}

Neste capítulo são elencados os critérios de inclusão para a presente revisão scoping sendo que serão explicitados quais os tipos de participantes, o conceito, o contexto e os tipos de estudos incluídos.

\subsection{Tipo de participantes}

A presente revisão scoping considerou todos os estudos que se foquem no uso da técnica ISBAR na transição dos cuidados de saúde em contexto hospitalar. Incluiu estudos com enfermeiros de todas as idades, generalistas e especialistas de todas as áreas que desenvolvam a sua atividade em contexto hospitalar.

\subsection{Conceito}

Esta revisão scoping considerou toda a pesquisa que aborda o tema da comunicação eficaz na transição dos cuidados de saúde, sendo a comunicação eficaz toda aquela que se caracteriza por ser oportuna, precisa, completa, sem ambiguidade, atempada e compreendida pelo recetor. Mais especificamente foram identificados estudos nos quais a técnica ISBAR é utilizada durante a transferência de informação sobre o doente. O ISBAR é uma ferramenta de padronização da comunicação em saúde que é reconhecida por promover a segurança do doente em situações de transição de cuidados (DGS, 2017).

\subsection{Contexto}

Nesta revisão scoping foram considerados todos os estudos que abordem o tema da comunicação eficaz na transição dos cuidados de saúde, particularmente os que utilizam a técnica ISBAR no contexto hospitalar. Todos os estudos fora do contexto hospitalar, ou que mencionem outras técnicas utilizadas na transferência de informação, foram excluídos.

\subsection{Tipos de estudos}

Esta revisão scoping considerou todos os estudos primários, quantitativos ou qualitativos, revisões sistemáticas da literatura, revisões da literatura, artigos de opinião relevantes de peritos da área e projetos. Se for encontrada duplicação de dados dos estudos primários nas revisões sistemáticas da literatura, nas revisões da literatura, será discutido, analisado e reportado de acordo com Peters et al., (2020).

Os estudos quantitativos que serão considerados para inclusão são os ensaios controlados randomizados, os ensaios controlados não randomizados, os estudos de coorte quase-experimentais, prospectivos e retrospectivos, os estudos de caso- 
controlo e os estudos descritivos. Estudos qualitativos incluindo desenhos fenomenológicos, etnográficos, grounded theory, estudos de caso e pesquisa histórica também serão considerados para inclusão.

As revisões sistemáticas de literatura incluem meta-análise e meta-síntese. As revisões de literatura incluem informação de investigação realizada, e conhecimento sobre o estado da arte de uma temática de forma resumida.

\subsection{Estratégia de pesquisa}

Relativamente à estratégia de pesquisa utilizada, a presente revisão scoping teve como finalidade identificar todos os estudos publicados nas bases eletrónicas CINAHL e MEDLINE. A escolha destas bases de dados relaciona-se com o facto de estas incluírem trabalhos científicos na área da saúde, nomeadamente na área da enfermagem.

A pesquisa foi levada a cabo sem limitação temporal dado que se pretendeu abranger toda a literatura existente sobre o tema em análise (Fallis, 2013; Peters et al., 2020). O estudo incluiu todos os estudos em português, espanhol e inglês. Artigos encontrados noutras línguas serão excluídos devido a falta de meios de tradução.

Uma estratégia em três etapas foi utilizada nesta revisão. Inicialmente foi conduzida uma pesquisa nas bases de dados MEDLINE (via PubMed) e CINAHL, seguida por uma análise do título e do resumo e dos termos indexados utilizados.

Em seguida, com todas as palavras - chave identificadas, foi realizada outra pesquisa em todas as bases de dados incluídas no estudo: JBI Database of Systematic Reviews and Implementation Reports, the Cochrane Library, Scopus, Science Direct, Epistemonikos e LILACS. Apesar de o termo ISBAR não ser um descritivo ao nível das ciências da saúde, a sua utilização foi essencial para direcionar a pesquisa especificamente para artigos relacionados com a técnica ISBAR.

A tabela 1 apresenta a estratégia de pesquisa e os resultados da segunda etapa.

Tabela 1 Resultados de Pesquisa

Pesquisa realizada nas bases de dados CINAHL e MEDLINE, utilizando os termos de pesquisa definidos e os resultados obtidos

\begin{tabular}{clcc}
\hline $\begin{array}{c}\text { Base de } \begin{array}{c}\text { codos } \\
\text { dados }\end{array} \\
\text { CINAHL }\end{array}$ & Termos de Pesquisa & $\begin{array}{c}\text { Número de } \\
\text { resultados }\end{array}$ & $\begin{array}{c}\text { Número de } \\
\text { Pesquisa }\end{array}$ \\
\cline { 2 - 4 } & ISBAR & 15 & S1 \\
\hline
\end{tabular}




\begin{tabular}{llcc}
\hline & Hospital & 249,952 & S3 \\
\cline { 2 - 4 } & (MM "Hand Off (Patient Safety)") OR "handoff" & 1,211 & $\begin{array}{c}\text { S4 OR S5 = } \\
\text { S6 }\end{array}$ \\
\hline Total & S1 AND S2 AND S3 AND S6 & S7 \\
\hline MEDLINE & ISBAR & 21 & S1 \\
\cline { 2 - 4 } & Nurs ${ }^{*}$ & 755,605 & S2 \\
\cline { 2 - 4 } & Hospital & $3,663,968$ & S3 \\
& Handoff & 1,067 & S4 \\
\hline Total & S1 AND S2 AND S3 AND S4 & S5 \\
\hline PCC (População - enfermeiros; Conceito - transferências, ISBAR; Contexto - Hospital) \\
\hline Fonte: Peters, Godfrey, Mclnerney, Munn, Tricco \& Khalil, (2020) & \multicolumn{2}{c}{}
\end{tabular}

$\mathrm{Na}$ terceira etapa foi realizada uma análise da lista de referências bibliográficas dos artigos identificados, de forma a identificar estudos adicionais (Fallis, 2013; Peters et al., 2020). Um estudo adicional foi integrado.

Os artigos pesquisados foram avaliados de acordo com a sua pertinência para a revisão, com a informação dada pelo título, pelo resumo e por dois revisores independentes. Nas situações em que através da leitura dos resumos, existiram dúvidas relativamente à relevância do estudo, o texto integral foi guardado. O texto integral foi considerado para o estudo quando todos os critérios de inclusão eram cumpridos.

Os revisores examinaram os textos integrais de forma separada, para que pudessem confirmar independentemente que todos os critérios de inclusão eram cumpridos pelos textos selecionados. Qualquer discordância que surgisse seria resolvida através da discussão dos procedimentos ou por intermédio de um terceiro revisor. Os dados foram extraídos sem discordância entre os revisores, seguindo as orientações de Levac, Colquhoun \& O'Brien, (2010). Estudos identificados a partir de listas de referências foram avaliados quanto à relevância.

\subsection{Apresentação de resultados}

A apresentação de resultados desta revisão scoping é baseada numa narrativa descritiva. A pesquisa decorreu entre maio e junho de 2019. Como demonstrado na figura 1, a procura identificou 9 potenciais estudos relevantes. $O$ número total de artigos obtidos foram 3 na base de dados CINAHL e 6 na base de dados MEDLINE. Da base de dados CINAHL foram selecionados 2 dos artigos encontrados, um foi excluído pelo resumo. Por sua vez, na base de dados MEDLINE, foram selecionados para esta revisão 4 artigos, um deles foi excluído por se encontrar duplicado e dois 
pela análise do resumo. Aos 5 textos integrais selecionados, juntaram-se outros 2 obtidos através da lista de referências dos estudos e recomendações dos especialistas dos restantes artigos. Um artigo foi excluído por não cumprir todos os critérios de inclusão após análise do texto integral. Finalmente, 6 estudos foram incluídos nesta revisão.

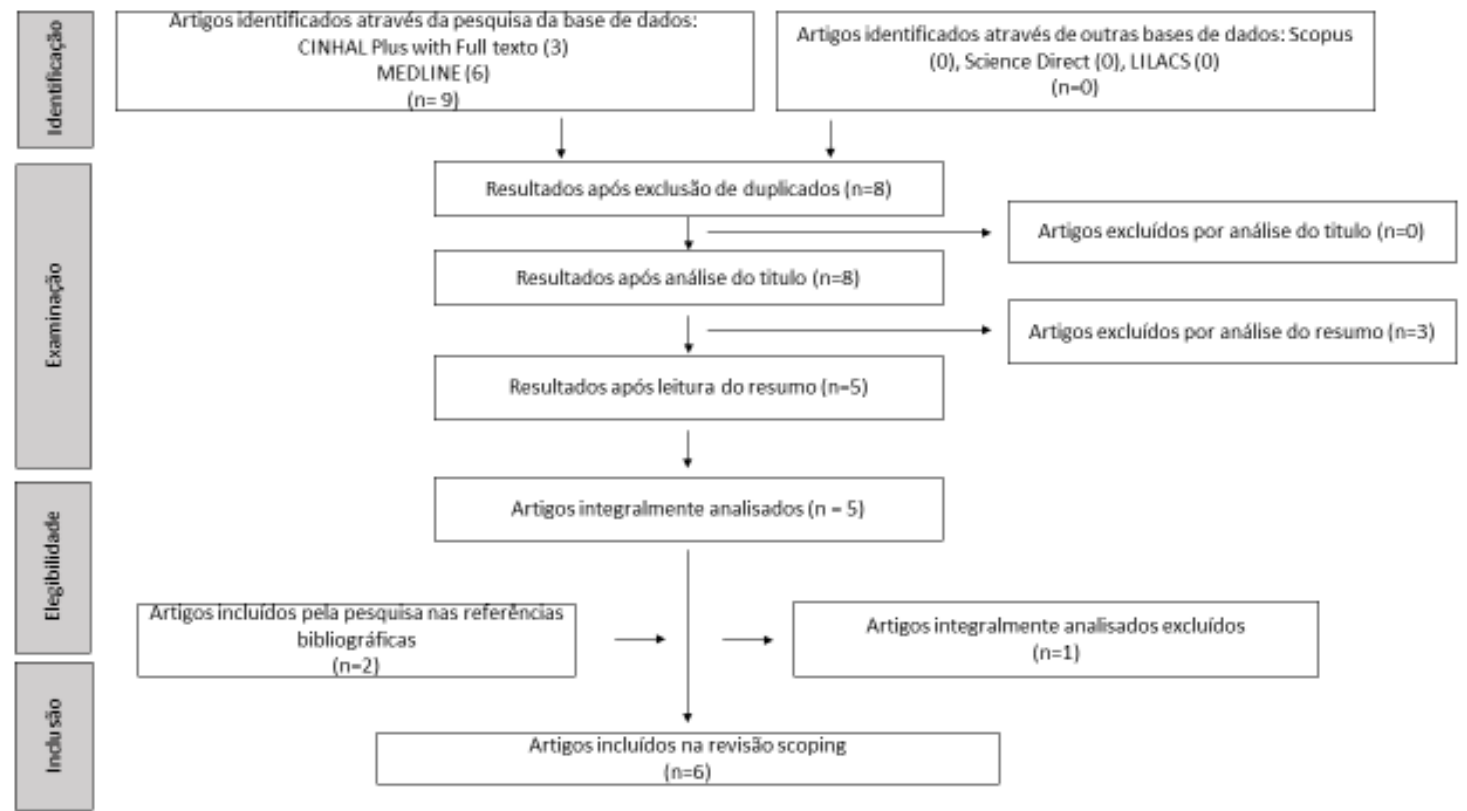

Figura 1. Diagrama de PRISMA referente ao processo de seleção dos estudos Fonte: Moher, Liberati, Tetzlaff, \& Altman, (2009)

Por forma a mapear os resultados dos 6 artigos incluídos nesta scoping review, elaborou-se uma tabela de extração de dados para permitir uma descrição sumária dos dados que estão em linha com o objetivo e tema deste presente estudo (Fallis, 2013; Peters et al., 2020).

O quadro elaborado para a extração de dados utilizado para a análise dos artigos, reúne a seguinte informação: a) Autor (es), b) Ano de Publicação, c) País de origem do estudo (onde foi realizado), d) Objetivos, e) População e tamanho da amostra (se aplicável), f) Metodologia/Método, g) Tipo de intervenção, comparação e detalhes de ambos (duração da intervenção) (se aplicável), h) duração da intervenção (se aplicável), i) Resultados e detalhes dos resultados (se aplicável), j) Achados principais relacionados com a questão da revisão scoping (Fallis, 2013; Peters et al., 2020). Posteriormente, aos dados relativos ao tipo de intervenção e resultados e detalhes dos resultados, realizou-se uma análise de conteúdo temática (Bardin, 2013) identificandose os temas ocorrência das transferências, problemas das transferências e benefícios da utilização do ISBAR. 
A extração de dados foi realizada por dois revisores, de forma independente.

Os estudos incluídos nesta scoping review foram levados a cabo na Austrália e em Itália. Em termos de pesquisa um dos estudos utilizou uma abordagem quantitativa e qualitativa (Mannix, Parry \& Roderick, 2017), dois estudos são projetos de melhoria da qualidade (Aldrich, Duggan, Lane, Nair, \& Hill, 2009; Mardegan; Heland; Whitelock; Millar \& Jones, 2013). Dois outros são estudos observacionais (Delupis, Mancini, Nota, \& Pisanelli, 2015; Spooner, Aiken, Corley, Fraser, \& Chaboyer, 2016) e o último é uma revisão sistemática da literatura. Os estudos incluídos foram publicados entre 2009 e 2017.

\section{DISCUSSÃO E CONCLUSÕES}

\subsection{Análise de resultados}

A presente revisão scoping sintetiza a evidência científica da literatura publicada nas bases de dados CINAHL e MEDLINE.

A evidência científica, identificada nas bases de dados supracitadas, demonstra que o tema da melhoria das transferências clínicas tem vindo a ganhar uma crescente valorização ao nível nacional e internacional, verificando-se a proliferação de literatura referente a melhorias na transferência clínica. As armadilhas da comunicação ineficaz estão bem documentadas na literatura, que inclui questões como o atraso no diagnóstico médico, eventos adversos ou tratamento ineficaz ou errado. Para além disso, as falhas de comunicação originam falta de confiança na relação entre o recetor e prestador de cuidados e situações de conflito, o que leva ao desaproveitamento de tempo e uma quebra nos processos da equipa multidisciplinar, colocando em risco a segurança do doente (Mannix et al., 2017).

No ano de 2007, a Comissão em Segurança e Qualidade nos serviços de Saúde na Austrália, iniciou um programa com a finalidade de melhorar as transferências clinicas, tendo recomendado a utilização da técnica ISBAR, nos momentos de transferência de informação. Estudos realizados demonstraram que a transferência de doentes tem vindo a ser reconhecida internacionalmente, como uma área de risco para a segurança do doente, podendo colocar em risco a sua vida, em especial no caso da pediatria (Mannix et al., 2017).

As transferências de informação ocorrem predominantemente durante as mudanças de turno de trabalho, ou quando os doentes são transferidos entre serviços ou hospitais. Em anos recentes a má prática durante as transferências foi identificada como sendo um dos fatores principais no dano causado ao doente. Cerca de $80 \%$ de erros médicos graves foram atribuídos a falhas de comunicação entre cuidadores, sendo que aproximadamente um em cada cinco doentes sofreram um evento adverso, 
resultante dessa falha na transferência de informação (Spooner et al., 2016). O estudo realizado por Mannix et al., demonstrou que procedimentos cruciais, como a transferência de doentes, podem ser melhorados utilizando uma abordagem que combine a segurança do doente e princípios de desenvolvimento da prática. Os achados deste estudo evidenciam benefícios específicos na existência de treino na transferência do doente. Os autores, previamente citados, concluíram que a baixa conformidade no uso da técnica ISBAR se devia, em parte, ao treino limitado na utilização da técnica. A utilização da técnica ISBAR assegura que a informação vital não é perdida e que a transferência de informação pode ser realizada de forma atempada, e eficiente, ao lado da cama do doente (Mannix et al., 2017). Neste estudo após a implementação da utilização da técnica ISBAR, verificou-se a existência de uma relação mais forte entre a compreensão dos participantes relativamente ao ISBAR e à sua aplicação durante as transferências de informação. Além disso, verificaram-se aumentos estatisticamente significativos nas melhores práticas de transferência, nomeadamente a inclusão familiar e verificações de segurança (Mannix et al., 2017).

Como previamente descrito, a comunicação ocorre em diferentes momentos e situações, como entre o pré-hospitalar e o serviço de urgência. Delupis et al. (2015), identificaram no seu estudo a existência de falta de padrões de comunicação quando os doentes eram transferidos entre o pré-hospitalar e o serviço de urgência. Identificaram também a inexistência de uma transferência formal de responsabilidades no cuidado do doente, com incongruências marcadas na informação transmitida. Neste estudo, somente os socorristas, que já tinham formação prévia no uso da técnica ISBAR, enunciaram 100\% dos itens da técnica (Delupis et al., 2015). Assim, é possível concluir através da evidência científica que o treino dos profissionais é um fator determinante para o correto uso da técnica ISBAR.

Estudos que abordam as transferências realizadas por enfermeiras foram também considerados, como o seguinte, que demonstra a importância da técnica ISBAR. Este estudo prospetivo observacional, realizado por Spooner et al. (2016), tem como tema, as transferências de informação realizadas pela equipa de enfermagem de um serviço de internamento de pediatria. As transferências foram gravadas em formato áudio durante mais de 20 dias. As gravações foram posteriormente transcritas e analisadas, utilizando análise de conteúdo dedutivo e indutivo. Foi determinada a frequência de conteúdo/ informação transmitida durante a transferência, que correspondem à técnica ISBAR. Existiram 40 transferências, resultantes de 277 doentes hospitalizados e uma média de 7 doentes discutidos em cada transferência. A maioria das enfermeiras transmitiu a identidade do doente (99\%), Situação (96\%) e antecedentes (88\%), no entanto, o conteúdo de avaliação (69\%) foi variado e as recomendações sobre o doente $(60 \%)$ foram discutidas menos frequentemente. Foi discutida uma ampla gama de informações adicionais que não se enquadram na técnica ISBAR. 
Estes achados indicam que a informação crítica do doente não foi transmitida de forma eficaz, o que pode colocar em causa a segurança do doente, sendo por isso importante implementar uma ferramenta de transmissão de informação para melhorar a transferência de informação e salvaguardar a segurança do doente (Spooner et al., 2016).

A transferência de doentes, utilizando uma comunicação eficaz, é essencial para assegurar uma gestão segura de todos os doentes e traz benefícios ao nível dos profissionais. O uso da técnica ISBAR possibilita, segundo a revisão sistemática realizada por Dawson, King \& Grantham (2013), nivelar a hierarquia dos profissionais e minimizar os pressupostos fornecendo expectativas comuns. Contudo, a utilização da técnica ISBAR é recomendado noutras situações, como em situações críticas. O Australian Resuscitation Council recomenda o uso da técnica ISBAR sendo que esta ferramenta permite transmitir informações durante um curto período de tempo, permanecendo os enfermeiros atentos à transmissão de informação. Também a Organização Mundial de Saúde corroborou o uso do ISBAR, que é reconhecido como sendo promotor da segurança dos doentes durante as transferências (Dawson et al., 2013).

Adotando estas recomendações, a utilização da técnica ISBAR tem vindo a aumentar, sendo que os autores Mardegan, Heland, Whitelock, Millar \& Jones (2013) levaram a cabo um projeto de melhoria da qualidade no Heidelberg Hospital na Austrália. O projeto consistia na elaboração de uma folha de transferência, baseada na técnica ISBAR, que serviria como guia para a transmissão da informação quando a equipa de emergência médica do hospital fosse ativada. Na avaliação deste projeto 58 (57.4\%) dos 101 entrevistados concordaram, ou concordaram fortemente, com a afirmação de que os pontos do ISBAR na parte superior do formulário facilitaram a transferência de informação (Mardegan et al., 2013).

Outro estudo, desenvolvido em dois hospitais australianos, demonstra que a utilização da técnica ISBAR melhorou a informação transmitida não só durante as mudanças de turno, mas também ao nível dos registos escritos (Aldrich et al., 2009). No mesmo estudo é descrito que os melhores elementos da técnica ISBAR são a sua simplicidade, portabilidade e memorabilidade. Esta técnica, engloba informações essenciais, requeridas pelos diferentes grupos profissionais. Por este motivo o ISBAR é multidisciplinar, permitindo uma melhor comunicação entre eles. Os participantes, dos dois estudos, referem de acordo com os autores, que a utilização de uma abordagem sistemática, aumentou as suas capacidades e a certeza sobre o estado de saúde do doente. Após a implementação do uso da técnica ISBAR a qualidade das transferências melhorou, os motivos pelos quais o doente estava a ser transferido, e a descrição do seu estado de saúde, ganharam simplicidade e clareza (Aldrich et al., 2009; Fallis, 2013). 
Assim, em consonância com o objetivo desta revisão scoping, foram analisados seis estudos que abordaram questões relacionadas com a utilização da técnica ISBAR e que respondem à questão de revisão. È possível afirmar que a utilização da técnica ISBAR durante a transferência de doentes, em diferentes situações, é positiva e várias vantagens foram identificadas.

\subsection{Limitações da revisão scoping}

A presente revisão scoping teve por base o protocolo da Joanna Briggs Institute (Peters et al., 2020). Durante a sua elaboração foram utilizados métodos de pesquisa claros tendo sido descritas todas as etapas da pesquisa. Porém, esta pesquisa teve por base a utilização o motor de busca EBSCOhost, selecionando as bases de dados CINAHL Plus full text e a MEDLINE with full text. A seleção de apenas duas bases de dados poderá ter limitado o número de artigos sendo essa uma limitação da presente revisão. A seleção dos termos indexados em língua inglesa poderá também ter limitado a pesquisa, bem como a seleção de artigos redigidos em língua Portuguesa, Inglesa e Espanhola.

\subsection{Conclusão}

A comunicação entre os profissionais de saúde é fundamental para promover a segurança dos clientes nas instituições hospitalares. A prática de cuidados de enfermagem tem momentos formais de transmissão da informação, que é uma das essências dos cuidados hospitalares. A transmissão de informação ambígua pode ter consequências ao nível dos diagnósticos, em eventos adversos, tais como erros, tratamentos pouco adequados ou ineficazes. Uma comunicação ineficaz pode também ser geradora de conflitos entre os profissionais de saúde.

Existem vantagens em utilizar ferramentas facilitadoras da comunicação como a técnica ISBAR, especificamente no contexto hospitalar. Esta técnica de comunicação, utilizada nos momentos de transferência, tem vindo a ser implementada em meio hospitalar. A sua utilização tem vantagens para os doentes e para a equipa de saúde, verificando-se um forte impacto ao nível da melhoria da segurança dos doentes durante as transferências clínicas. Apresenta também vantagens para os profissionais de saúde pela sua simplicidade, clareza de conteúdos, portabilidade e memorabilidade.

A utilização da técnica ISBAR, pelas vantagens demonstradas vai reforçar a importância que a comunicação eficaz tem na segurança dos doentes com consequentes melhorias dos resultados. O ISBAR deverá ser um instrumento diário de comunicação, com uma linguagem global, simples e eficiente dos cuidados de saúde. Para além destas vantagens, esta revisão de literatura permitiu também compreender 
a importância da realização de formação aos profissionais de forma a garantir a utilização correta da técnica ISBAR.

\subsection{Implicações para a prática e para a investigação}

Relativamente às implicações para a prática, o uso da técnica ISBAR é relativamente recente sendo que, em Portugal, só em 2017 foi recomendada a sua utilização através de uma norma de orientação clínica, a NOC 001/2017. Assim, é importante o desenvolvimento e implementação de estratégias e programas, que incentivem a sua utilização, nos contextos de saúde Portugueses, permitindo assim uma operacionalização da NOC 001/2017. Esta revisão vem destacar algumas das vantagens que podem advir do uso da técnica ISBAR durante a transferência de doentes, reforçando a importância e pertinência da sua utilização. Contudo esta revisão permitiu também evidenciar a importância da formação, tendo sido identificados dois estudos, através dos quais foi possível compreender que profissionais com treino utilizam de modo eficaz a técnica. Assim, uma das estratégias para uma utilização eficaz da técnica é a realização de formação aos profissionais de saúde de forma sistematizada e continua.

Ao nível das implicações para a investigação, é essencial o desenvolvimento de estudos que possibilitem identificar vantagens e desvantagens na utilização da técnica ISBAR, no contexto específico Português, onde existem características económicas e demográficas específicas. Ainda de referir que a técnica de análise de conteúdo foi imprescindível para a qualidade da scoping review.

\section{Referências}

Aldrich, R., Duggan, A., Lane, K., Nair, K. \& Hill, K. (2009). ISBAR revisited: Identifying and Solving BARriers to effective clinical handover in inter-hospital transfer: Final Project Report on Pilot Study. Newcastle Hunter New England Health.

Bardin, L. (2013). Análise de Conteúdo. Lisboa: Edições 70.

Dawson, S., King, L., \& Grantham, H. (2013). Review article: Improving the hospital clinical handover between paramedics and emergency department staff in the deteriorating patient. Emergency Medicine Australasia: EMA, 25(5), 393-405. Doi:10.1111/17426723.12120 .

Delupis, F. D. D., Mancini, N., Nota, T \& Pisanelli, P. (2015). Pre-hospital/emergency department handover in Italy. Internal And Emergency Medicine, 10(1), 63-72. Doi:10.1007/s11739-014-1136-x.

Despacho n. $.01400-\mathrm{a} / 2015$ n. .28 de 10 de Fevereiro (2015). Plano Nacional para a Segurança dos Doentes 2015-2020. Diário da República, 2. ${ }^{2}$ série. Ministério da Saúde. 
Direção Geral da Saúde (2011). Avaliação da Cultura de Segurança do Doente numa amostra de Hospitais Portugueses. Resultados do Estudo Piloto. Lisboa.

Direção Geral da Saúde (2017). Comunicação eficaz na transição de cuidados de saúde. Norma no001/2017 de 08 de Fevereiro. Departamento da Qualidade na Saúde. Direção Geral da Saúde, 1-8.

Eppich, W. (2015). "Speaking Up" for Patient Safety in the Pediatric Emergency Department. Elsevier. 16; 1522-8401.

Fallis, A. (2013) Reviewer's Manual. Journal of Chemical Information and Modeling 53(9):1689_ 99.

Figueiredo, A. R, Potra, T. \& Lucas, P. (2019) Comunicação eficaz nas transições de saúde: um revisão scoping in Costa, A. P, Oliveira, C., Synthia, E., Ribeiro, J., Presado, H. \& Baixinho, C (Ed.) (2019) CIAIQ2019 - Atas do 8o congresso ibero-americano em investigação qualitativa - investigação qualitativa em saúde. V.2. ISBN: 978-989-544764-0.

Funk, E., Taicher, B., Thompson, J., lannello, K., Morgan, B. \& Hawks, S. (2016) Structured Handover in the Pediatric Postanesthesia Care Unit. Journal of Perianesthesia Nursing, 31(1):63-72.

Institute of Medicine. (2000) To Err is Human: Building a Safer Health System. Washington, D.C.: IOM.

Levac, D., Colquhoun, H. \& O'Brien, K. K. (2010). Scoping studies: advancing the methodology. Implement Science; 5(69):1-9.

Mannix, T., Parry, Y., \& Roderick, A. (2017). Improving clinical handover in a pediatric ward: implications for nursing management. Journal of Nursing Management, Doi:10.1111/jonm.12462.

Mardegan, K., Heland, M., Whitelock, T., Millar, R., \& Jones, D. (2013). Developing a Medical Emergency Team Running Sheet to Improve Clinical Handoff and Documentation. Joint Commission Journal On Quality \& Patient Safety, 39(12), 570-575.

Martin, H., \& Ciurzynski, S. (2015). Situation, Background, Assessment, and Recommendation Guided Huddles Improve Communication and Teamwork in the Emergency Department. Journal of Emergency Nursing, 41 (6) 484-488.

Moher, D., Liberati, A., Tetzlaff, J. \& Altman, D. G. (2009). Preferred Reporting Items for Systematic Reviews and Meta-Analyses: The PRISMA Statement. Annals of Internal Medicine; 151:264-269. Doi: 10.7326/0003-4819-151-4-200908180-00135.

Peters, M. D. J., Godfrey, C. M., Khalil, H., Mclnerney, P., Parker, D. \& Soares, C. B. (2015). Guidance for conducting systematic scoping reviews. International Journal of Evidence Based Healthcare; 13(3):141-6. 
Peters, M. D. J., Godfrey, C., McInerney, P., Munn, Z., Tricco, A. C. \& Khalil, H. (2020) Chapter 11: Scoping Reviews. In: Aromataris E, Munn Z (Editors). Joanna Briggs Institute Reviewer's Manual, JBI (2020). Available from https://reviewersmanual.joannabriggs.org/

Shapiro, J. (2017) SBAR: A Better Way to Communicate. Practice Perfect. January 20 (Pediatry Management): 41-2.

Silva, E. M. B., Pedrosa, D. L. L., Leça, A. P. C., \& Silva, D. M. (2016). Perceção dos profissionais de saúde sobre a cultura de segurança do doente pediátrico. Revista de Enfermagem Referência, 4 (9), 87-95. http://dx.doi.org/10.12707/RIV16007.

Spooner, A., Aitken, L., Corley, A., Fraser, J., \& Chaboyer, W. (2016). Nursing team leader handover in the intensive care unit contains diverse and inconsistent content: An observational study. International Journal Of Nursing Studies, 61165-172. Doi:10.1016/j.jijnurstu.2016.05.006. 